\title{
Changes produced in the intramuscular tissue via dry needling measured with magnetic resonance imaging
}

\author{
Baraja-Vegas L. ${ }^{1}$ Piqueras-Sanchiz P. ${ }^{2} \quad$ Bautista I.J. ${ }^{3}$ Martín Rodríguez S. ${ }^{4}$ \\ ${ }^{1}$ Universidad Católica de Valencia, Valencia, Spain \\ 2 Sport Plus Center, Sevilla, Spain \\ 3 Fisiosalud Elite, Health, Training \& Innovation, Universidad de \\ Granada, Granada, Spain \\ ${ }^{4}$ University of Verona, Italy \\ Rev Fisioter Invasiva 2019;2:69.
}

\begin{abstract}
Keywords

- dry needling

- tissue edema

- medial gastrocnemius

- MRI

Introduction Myofascial trigger points are a common cause of pain and clinically observed local muscle sensitivity. The improvement associated with the muscle relaxation effect (reduction of muscle rigidity) produced by dry needling (DN) is still not well known. Thus, it is thought that the regeneration of the tissue that has been destructed by dry needling via the inflammatory process occurs with the return to normal muscle contractility.

Aims The aim of this study was to identify whether the local inflammatory effect is immediately induced by DN, using magnetic resonance imaging (MRI).

Material and Methods 18 asymptomatic patients were evaluated, with latent trigger points in the medial gastrocnemius (MG). We used an intergroup research design to study differences in MRI signal in the medial gastrocnemius, before and after performing DN. The MG which presented the most pain in response to pressure, was used as the experimental or intervention group, whereas the contralateral MG was used as the control group. MRI was used with signal intensity (STIR), to identify signal changes due to local inflammation. An ANOVA test was performed to analyze the influence of DN in the STIR variable and a Student's t-test for dependent samples was used to compare the perception of pain after DN.

Results The STIR increased by $128.97 \%$ after DN in the experimental group. The effect of the interaction showed significant differences $(F(1.34)=235, p=0.0001, r=0.93)$. The Bonferroni post hoc tests showed significant differences [differences in means and $95 \%$ confidence interval $(95 \% \mathrm{Cl})=198(172-224)]$ signal intensity $(F(1.34)=236$, $p=0.001 ; r=0.93 ; d=5.03)$. Furthermore, a significant group was found (control vs. experimental group) after controlling for the effects of the intervention ( $F$ $(1.33)=9.95, p=0.003, r=0.48, d=1.10)$. Regarding the perception of pain in response to pressure, a significant reduction was found between the pre and post intervention measurements $(t(17)=12.40, p=0.001, r=0.65, d=1.71)$. The Pearson's correlation coefficient did not display any correlation among any variable.

Conclusion Intramuscular edema appears immediately after (1h) the application of $\mathrm{DN}$, which indicates an inflammatory process with an unexpected reduction in pain perception. Our results are in line with other findings in mice which display signs of an inflammatory response after DN. A limitation of this study was the lack of further MRI measurements to detect when the edema resolved.
\end{abstract}

DOI https://doi.org/ 10.1055/s-0039-3401890. ISSN 2386-4591.
Copyright $\odot 2019$ by Thieme Revinter Publicações Ltda, Rio de Janeiro, Brazil 